\title{
Vitamin A deficiency and child feeding in Beijing and Guizhou, China
}

\author{
Jing-Xiong Jiang, Liang-Ming Lin, Guang-Li Lian, Ted Greiner \\ Beijing, China and Washington, USA
}

Background: Vitamin A deficiency (VAD) is one of the three major micronutrient deficiencies in the world. In order to investigate the status of VAD and child feeding in China, we conducted the survey in Beijing city and Guizhou province.

Methods: We included a high socioeconomic area (Beijing) and a low socioeconomic area (Guizhou province) in China in our study. Participants included 1236 randomly selected children aged 0-71 months from stratified clusters (628 in Beijing and 608 in Guizhou), 409 from urban and 827 from rural areas. A food intake frequency questionnaire was used for dietary assessment. Fluorescence microanalysis was carried out to measure serum retinol concentrations. Serum retinol cut-off values of less than $20 \mu \mathrm{g} / \mathrm{dl}$ and $30 \mu \mathrm{g} / \mathrm{dl}$ were defined as sub-clinical VAD and suspected sub-clinical VAD, respectively.

Results: No xerophthalmia or night blindness was found. The mean concentration of serum retinol was $31.5 \mu \mathrm{g} / \mathrm{dl}$ in the high socioeconomic group, and $\mathbf{2 6 . 5}$ $\mu \mathrm{g} / \mathrm{dl}$ in the low socioeconomic group. Rural infants had lower concentrations of serum retinol compared with the urban ones $(26.9 \pm 8.1 \mu \mathrm{g} / \mathrm{dl}$ vs $31.8 \pm 7.3 \mu \mathrm{g} / \mathrm{dl})$. The prevalence of sub-clinical VAD among all the children was $7.8 \%$, and increased to $15.7 \%$ in children from the low socioeconomic group. In infants from the high socioeconomic area, the prevalence of suspected subclinical VAD was $\mathbf{3 8 . 0} \%$, increasing to $\mathbf{5 9 . 5 \%}$ in infants from the low socioeconomic area. The children from the low socioeconomic area had significantly lower frequency of intake of meat than the children in other groups. The prevalence of suspected sub-clinical VAD was higher in the children with lower consumption frequency of vitamin A rich foods than the children with higher consumption frequency of vitamin A rich foods.

Author Affiliations: National Center for Women's and Children's Health, Beijing, China (Jiang JX, Lian GL); Capital Institute of Pediatrics, Beijing, China (Lin LM); PATH, Washington DC, USA (Greiner T)

Corresponding Author: Jing-Xiong Jiang, National Center for Women's and Children's Health, Beijing 100013, China (Tel: 86-10-64484396; Fax: 86-10-64296782; Email: jiangjingxiong@chinawch.org.cn)

(C)2008, World J Pediatr. All rights reserved.
Conclusions: VAD appears to be a moderate public health problem in certain areas of China. In areas with low socioeconomic status, VAD in childrean is more severe, and infants may be the group at the highest risk for VAD. Inadequate intake of vitamin A rich foods may result in VAD. A comprehensive long-term national strategy needs to be fostered in China for the treatment and prevention of the deficiency.

World J Pediatr 2008;4(1):20-25

Key words: infants;

feeding and eating disorders; socioeconomic status; vitamin A deficiency

\section{Introduction}

T 7 itamin A deficiency (VAD) is one of the world's three major micronutrient problems. The first apparent clinical warning signs of VAD are night blindness and xerophthalmia. Although the number of clinically affected children has declined in recent years with many interventional programs, the number of children with sub-clinical VAD is still huge in the world. Sub-clinical VAD increases children's susceptibility to infection, reduces physical growth, and decreases the possibility of survival from serious illness. Several epidemiological studies have identified a relationship between sub-clinical VAD and a higher rate of mortality. ${ }^{[1-3]}$

Adequate vitamin A intake in children depends on suitable feeding, and various feeding problems can increase the risk for VAD. The infant feeding process can be divided into different stages, each is associated with patterns of feeding and interaction between mother and child. ${ }^{[4]}$ Some patterns that lead to an increased risk of VAD are lack of exclusive breastfeeding to the infant, lack of preparation of special complementary foods, and relatively late introduction of solid foods. Many studies have investigated an association between xerophthalmia, a clinical sign of VAD, and food intake. ${ }^{[5]}$ Sub-clinical VAD is also associated with a low total intake of vitamin $A .^{[6]}$ Most studies examining dietary patterns 
of children with night blindness have found significant differences in consumption of preformed vitamin A and provitamin A foods. A significantly reduced risk of xerophthalmia was found in children whose mothers reported feeding them vitamin A-rich foods regularly during the first year of life. ${ }^{[7]}$ Increased consumption of dietary vitamin A has been repeatedly mentioned as a long-term solution to the problem of VAD.

VAD is a public health problem in many developing countries. It is estimated that approximately 140 million preschool-aged children in the world suffer from VAD every year and another 4.4 million children suffer from xerophthalmia ${ }^{[8,9]}$ Nearly half of all cases of VAD and xerophthalmia occur in South and Southeast Asia. Even in some developed countries such as the United States, young children are also susceptible to VAD if their dietary intake of the essential vitamins is inadequate. ${ }^{[10]}$ Protein-energy malnutrition has decreased obviously in children in China recently, however, the prevalence of micronutrient deficiencies has received little attention. In order to obtain information on vitamin A status and identify possible intervention strategies, a survey was conducted from November 1999 to January 2000. Here we present the results from a high socioeconomical area, Beijing city, and a relatively low socioeconomic area, Guizhou province.

\section{Methods \\ Subjects}

According to the Classification of Chinese Health and Economic Status, ${ }^{[1]}$ six socioeconomic classes of community are defined for each city or county in China. The first-class is the area with high socioeconomic level (richest county) and the sixth-class is the area with very low socioeconomic level (poorest county). In Beijing, one big city (Beijing city), one first-class county (Daxing county) and one second-class county (Yanqing county) were selected in the survey. In Guizhou province, one middle city (Zunyi city), one third-class county (Puding county) and one fourth-class county (Majing county) were selected in the survey. Thus, our samples included each of the six classes of community. We defined Beijing city as the high socioeconomic urban group and the middle city in Guizhou province as the low socioeconomic urban group. The first- and second-class counties in Beijing were defined as high socioeconomic rural group and the third- and fourthclass counties in Guizhou province were defined as the low socioeconomic rural group.

The participants in the study included 1236 children aged 0-71 months (628 in Beijing and 608 in Guizhou), $409(33 \%)$ in the urban areas, and $827(67 \%)$ in the rural areas. In each city studied, two districts were selected randomly and, from each of them, three communities were selected randomly. In each county, three towns were selected randomly. In each town, two villages were selected randomly. Three age groups were defined in the sample: 0-23 months, 24-47 months and 48-71 months. In each community or village, 11-12 children were selected randomly from a list of all children for each age group by the village doctors in the rural areas and the community health doctors in the urban areas.

\section{Study methods}

A questionnaire was developed based on the Helen Keller International Food Frequency Method ${ }^{[12]}$ to obtain information about consumption frequency of vitamin A dietary and meat intake in a week before the interview. The pre-tested questionnaire was administered by trained interviewers at each site. The interview was performed by two trained provincial doctors and one central personnel in each field site. In all households, the primary respondent was the mother of the selected child or the caregiver who knew the child best. A $200 \mu \mathrm{l}$ blood sample was obtained from each child by finger puncture. Blood samples were protected from light, stored in cool boxes and centrifuged for 10 minutes within 4-6 hours after collection. The serum was protected from light and stored at $-20^{\circ} \mathrm{C}$ for up to 9 days. After the field work was finished, the serum samples were transported for up to 6 hours in a cool box to the Capital Institute of Pediatrics in Beijing, where they were stored at $-70^{\circ} \mathrm{C}$ before analysis, which took place within three months.

\section{Measurement of serum retinol}

Serum samples were analyzed for retinol at the Capital Institute of Pediatrics in Beijing using fluorescence microanalysis under a strict quality-control protocol with a fluorescence spectrophotometer (RF-540, Japan). The test environment, test method, equipment settings, reagent preparation and protection, test procedure, result calculation and data coding followed the standards established by the Centers for Disease Control (Atlanta, USA). Two high concentration serum standards and two low concentration serum standards were tested before and after testing every day. If any of the average value or range for the standard serum was out of $99 \%$ confidence intervals (CI), all the tests performed on that day should be repeated. The coefficient of variation (cv) for the various runs varied from $2.9 \%$ to $9.9 \%$.

Before testing, 100 serum samples from the Centers for Diseases Control (Atlanta, USA) were tested as training practice. The defined concentration of vitamin A for these samples was $38.16 \mu \mathrm{g} / \mathrm{dl}$. The reference values were $35-45 \mu \mathrm{g} / \mathrm{dl}$. The results obtained at the 
Capital Institute of Pediatrics were 36.5-44.1 $\mu \mathrm{g} / \mathrm{dl}$.

The definition of VAD established by the Chinese Pediatric Society ${ }^{[13]}$ was used in this report: clinical VAD was defined as serum retinol $<10 \mu \mathrm{g} / \mathrm{dl}$, subclinical VAD $10-<20 \mu \mathrm{g} / \mathrm{dl}$, and suspected sub-clinical VAD $20-<30 \mu \mathrm{g} / \mathrm{dl}$.

\section{Data analysis}

The Statistical Program for Social Sciences (SPSS) was used to analyze the data. The criterion for statistical significance was set at $P<0.05$. Differences between study groups were assessed by the Chi-square test, independent sample $t$ test and analysis of variance (ANOVA) for categorical and continuous variables, respectively. Linear correlation was also used in this paper.

\section{Results}

\section{Vitamin A status in children}

No xerophthalmia or night blindness was found. The overall mean serum retinol concentration was $31.5 \mu \mathrm{g} /$ dl. Rural children had significantly lower serum retinol concentrations than urban children. The serum retinol concentration in the low socioeconomic group was significantly lower than that in the high socioeconomic group, and the difference was greater in the rural areas. The family income varied in groups with different socioeconomic status (Table 1). There was a statistically significant positive linear correlation between serum retinol and family income $(r=0.239, P<0.01)$.

There were significant differences between areas by both cut-offs of $20 \mu \mathrm{g} / \mathrm{dl}$ and $30 \mu \mathrm{g} / \mathrm{dl}$. Sub-clinical VAD (serum retinol $<20 \mu \mathrm{g} / \mathrm{dl}$ ) was found in $7.8 \%$ of all the examined children $(10.9 \%$ of rural and $4.9 \%$ of urban children). The prevalence of suspected sub-clinical VAD (serum retinol $<30 \mu \mathrm{g} / \mathrm{dl}$ ) was $38.0 \%$ in all the children. In rural children of the low socioeconomic group, the prevalence was between $47.9 \%$ and $66.6 \%$, depending on the age group. The prevalence varied from $16.8 \%$ to $52.4 \%$ among different socioeconomic groups, and the differences were statistically significant.

Infants had a significantly lower serum retinol concentration than older children, both in the urban and rural areas (Table 2). There was a statistically significant positive linear correlation between serum retinol and age $(r=0.132, P<0.01)$. Infants had significantly higher

Table 1. Vitamin A status and family income in different socio-economic groups

\begin{tabular}{|c|c|c|c|c|}
\hline \multirow{2}{*}{ Variables } & \multicolumn{2}{|l|}{ Urban areas } & \multicolumn{2}{|l|}{ Rural areas } \\
\hline & High $(n=202)$ & Low $(n=207)$ & High $(n=426)$ & Low $(n=401)$ \\
\hline Serum retinol $(\mu \mathrm{g} / \mathrm{dl})($ mean $\pm \mathrm{SD})$ & $37.5 \pm 7.9$ & $35.6 \pm 7.8$ & $31.6 \pm 8.3$ & $26.5 \pm 8.1^{*}$ \\
\hline Prevalence of sub-clinical VAD (\%) & 2.0 & 1.4 & 6.3 & $15.7^{\dagger}$ \\
\hline Prevalence of suspected sub-clinical VAD (\%) & 16.8 & $23.2^{\dagger}$ & 41.8 & $52.4^{\dagger}$ \\
\hline Family income in $1998(\mathrm{RMB} 1000)($ mean \pm SD) & $87.1 \pm 65.0$ & $45.3 \pm 26.1$ & $8.0 \pm 5.7$ & $1.8 \pm 1.4^{*}$ \\
\hline
\end{tabular}

*: comparing the four socio-economic groups by ANOVA, $P<0.05$; $\uparrow$ : comparing the high and low socio-economic groups in either urban or rural areas by the Chi-square test, $P<0.05$. High: high socio-economic group; Low: low socio-economic group.

Table 2. Serum retinol concentration and prevalence of VAD in the children by age

\begin{tabular}{|c|c|c|c|c|}
\hline \multirow[b]{2}{*}{ Age (mon) } & \multicolumn{2}{|l|}{ Urban areas } & \multicolumn{2}{|l|}{ Rural areas } \\
\hline & $\begin{array}{l}\text { Serum retinol }(\mu \mathrm{g} / \mathrm{dl}) \\
(\text { mean } \pm \mathrm{SD})\end{array}$ & $\begin{array}{c}\text { Prevalence of suspected } \\
\text { sub-clinical VAD (\%) }\end{array}$ & $\begin{array}{l}\text { Serum retinol }(\mu \mathrm{g} / \mathrm{dl}) \\
(\text { mean } \pm \mathrm{SD})\end{array}$ & $\begin{array}{l}\text { Prevalence of suspected } \\
\text { sub-clinical VAD (\%) }\end{array}$ \\
\hline$<12$ & $31.8 \pm 7.3^{*}$ & $41.2^{\dagger}$ & $26.9 \pm 8.1^{*}$ & $59.5^{\dagger}$ \\
\hline $12-23$ & $37.5 \pm 6.9$ & 34.7 & $27.3 \pm 7.5$ & 43.5 \\
\hline $24-35$ & $35.9 \pm 7.6$ & 33.3 & $28.7 \pm 8.2$ & 40.2 \\
\hline $36-71$ & $37.9 \pm 7.8$ & 31.5 & $30.2 \pm 8.2$ & 38.5 \\
\hline
\end{tabular}

*: comparing the four age groups by ANOVA, $P<0.05$; $\uparrow$ : comparing infants and older children by the Student's $t$ test, $P<0.05$.

Table 3. Percentage of children who consumed selected foods twice or more times in different groups

\begin{tabular}{|c|c|c|c|c|}
\hline \multirow{2}{*}{ Selected foods } & \multicolumn{2}{|l|}{ Urban areas } & \multicolumn{2}{|l|}{ Rural areas } \\
\hline & High $(n=202)$ & Low $(n=207)$ & High $(n=426)$ & Low $(n=401)$ \\
\hline Milk & 85 & $76^{*}$ & 58 & $4^{*}$ \\
\hline Meat & 91 & $81^{*}$ & 68 & $4^{*}$ \\
\hline Fish and seafood & 49 & $24^{*}$ & 14 & $5^{*}$ \\
\hline Liver & 16 & 23 & 6 & $3^{*}$ \\
\hline DGLV and YOV & 97 & $89^{*}$ & 92 & 91 \\
\hline Eggs & 92 & $76^{*}$ & 85 & $17^{*}$ \\
\hline
\end{tabular}

*: comparing high and low socio-economic groups by the Chi-square test, $P<0.05$. High: high socio-economic group; Low: low socio-economic group. DGLV: dark green leafy vegetables; YOV: yellow/orange vegetables. 
Table 4. Percentage of low serum retinol level $(<30 \mu \mathrm{g} / \mathrm{dl})$ in children by age and intake frequency of various foods

\begin{tabular}{|c|c|c|c|c|}
\hline Consumption frequency & $<12$ mon $(n=164)$ & $12-23$ mon $(n=226)$ & $24-35$ mon $(n=221)$ & $36-71$ mon $(n=625)$ \\
\hline \multicolumn{5}{|l|}{ Animal milk } \\
\hline High & $36^{*}$ & $28^{*}$ & $27^{*}$ & $28^{*}$ \\
\hline Low & 80 & 77 & 67 & 53 \\
\hline \multicolumn{5}{|l|}{ Animal milk } \\
\hline High & $33^{*}$ & 36 & 52 & 32 \\
\hline Low & 65 & 51 & 46 & 43 \\
\hline \multicolumn{5}{|l|}{ DGLV and YOV } \\
\hline High & $52^{*}$ & 49 & 47 & 41 \\
\hline Low & 77 & 44 & $17^{\dagger}$ & 56 \\
\hline \multicolumn{5}{|l|}{ Eggs } \\
\hline High & $41^{*}$ & $39^{*}$ & $39^{*}$ & $33^{*}$ \\
\hline Low & 83 & 72 & 62 & 56 \\
\hline \multicolumn{5}{|l|}{ Meat } \\
\hline High & $47^{*}$ & 46 & $41^{*}$ & $36^{*}$ \\
\hline Low & 71 & 55 & 61 & 56 \\
\hline \multicolumn{5}{|l|}{ Fish and seafood } \\
\hline High & 42 & $18^{*}$ & $21^{*}$ & $24^{*}$ \\
\hline Low & 65 & 57 & 51 & 46 \\
\hline
\end{tabular}

*: comparing high and low consumption frequency of the food in each age group by the Chi-square test, $P<0.05$; $†$ : six $24-35.9$ months old children with low DGLV/YOV intake and only one of them had low serum retinol. High: $\geq$ twice/week; Low: < twice/week. DGLV: dark green leafy vegetables; YOV: yellow/orange vegetables.

prevalence of sub-clinical VAD than the older children.

\section{Dietary intake in children}

Table 3 shows the proportion of the children who consumed various relevant types of foods twice or more per week. The children in the low socioeconomic group in the rural area had a significantly lower frequency of consumption of meat than the children in other groups.

\section{Food intake and vitamin A status in different age groups}

The relationship between diet and vitamin A status is shown in Table 4. Dividing intake into those above and below or equal to once a week for each of the selected vitamin A rich foods, the prevalence of suspected subclinical VAD was higher in the children with a low frequency of intake of all foods in almost all age groups. Among all the children, lower intake frequency (once a week or none) was more relevant to a higher risk of having suspected VAD $(<30 \mu \mathrm{g} / \mathrm{dl})$ than high food intake frequency (every day or 2-3 times a week). At the age of 12-23 months, for instance, children with a low frequency of intake of eggs had a 2.2-fold higher risk of susected sub-clinical VAD than those with a high frequency $(95 \%$ CI 1.5, 3.3).

\section{Discussion}

\section{VAD in Beijing and Guizhou}

We examined a subset of the data of Beijing and
Guizhou province obtained from a national vitamin A survey, which was conducted in 13 provinces and one municipality. The results of this national survey showed that the prevalence of sub-clinical VAD in children was $11.7 \%$ and suspected sub-clinical VAD was $39.2 \% .{ }^{[14]}$ According to the Classification of Chinese Health and Economic Status, ${ }^{[1]}$ Beijing is the area with high socioeconomic level and Guizhou is low in both urban and rural areas. Among 82 counties in Guizhou province, 70 are poor or very poor counties. There are no poor or very poor counties in Beijing.

The data of serum retinol distributions were used to assess the extent of VAD in children. According to the definitions in China and abroad, ${ }^{[8,9,12,15]} 6.9 \%$ of the children had sub-clinical VAD and $33.7 \%$ had suspected sub-clinical VAD. The mean value of serum retinol was $31.5 \mu \mathrm{g} / \mathrm{dl}$. The prevalence of sub-clinical VAD was $15.7 \%$ in the low socioeconomic group. $\mathrm{WHO}^{[16,17]}$ suggested that the prevalence of sub-clinical VAD at $10 \%-<20 \%$ indicated a moderate public health issue. However, the XX International Vitamin A Consultative Group Meeting suggested $15 \%$ as a severe public health problem. ${ }^{[18]}$ Among the children in rural low socioeconomic group, the prevalence of sub-clinical VAD was $15.7 \%$ and fit the defined criteria of severe health problem. There were significant differences in the prevalence of VAD in different socioeconomic areas, with moderate to severe public health problem in the low socioeconomic areas.

$33.8 \%$ of children in urban areas and $42.4 \%$ of children in rural areas had serum retinol concentrations 
less than $30 \mu \mathrm{g} / \mathrm{dl}$, which is considered at risk of $\mathrm{VAD},{ }^{[19,20]}$ and the prevalence of the deficiency was significantly higher in the children in low socioeconomic group. In the children under 3 years old, almost twothirds were affected by subnormal concentrations of serum retinol. Since no previous data were available for vitamin A status in these areas, we could not make any comparisons over time. A statistically significant positive linear correlation between serum retinol and family income was found, as found in other countries. ${ }^{[21]}$ Thus, it appears that many more children with potentially suboptimal concentrations of serum retinol were undisclosed except the children with VAD, especially in young children in rural areas with low socioeconomic status.

\section{High risk groups for VAD}

The prevalence of VAD in children increases with age. ${ }^{[22,23]}$ Typically infants are expected to have a higher vitamin A status due to breastfeeding. ${ }^{[15]}$ But in this survey, we found the opposite results, which might be due to considering the county as a whole. While too little is known about the normal levels of retinol in healthy infants, it would appear that they have the highest prevalence of subclinical VAD and suspected subclinical VAD among all the age groups in this study. VAD is associated with unsuitable feeding in infants, ${ }^{[1,24]}$ which can be influenced by maternal nutritional knowledge and socioeconomic status. ${ }^{[4,5]}$ In this survey, we found that the low socioeconomic status was associated with low vitamin A status. However, it is not clear if VAD is also influenced by poor maternal feeding knowledge. Breast milk is the most important source of vitamin A for infants. Improved maternal vitamin A status will increase vitamin A concentration in breast milk. In this survey, only $19.5 \%$ of infants $(<12$ months) were predominantly breastfed (Questions were not asked to mothers, but in a way that allowed the prevalence of exclusive breastfeeding to be estimated), $55.5 \%$ of infants were partially breastfed, and $22.0 \%$ were not breastfed at the time of the interview. Strictly defined, the duration of exclusive breastfeeding appears to be short in China. Sweetened water, supplemental milks and other fluids were commonly given in the early weeks of life in infants, which may explain the low vitamin A levels seen in these infants, even when they were breastfed.

\section{Dietary intake and VAD in children}

Poverty is associated with inadequate food intake which in turn leads to poor vitamin A status in children. ${ }^{[25]}$ Foods of animal origin are rarely given to children in low socioeconomic areas. Even though consumption of green-leaf vegetables and yellow fruits was very high in these areas, the mean serum retinol concentration was low and the prevalence of VAD was high. In developing countries, $27 \mu \mathrm{g}$ of $\beta$-carotene is taken from green-leaf vegetables to yield $1 \mu \mathrm{g}$ of retinol. ${ }^{[9]}$ Hence, it is difficult for young children to consume sufficient quantities of vegetables if vegetables are the only source of $\beta$-carotene/ vitamin A. In our survey, children in each age group with low intake frequency of vitamin A rich foods had a higher prevalence of suspected sub-clinical VAD. Vitamin A supplementation and fortification were rare in China at the time of the survey, thus food intake was a very important factor for VAD. Direct cause or effect between consumption of each type of foods and vitamin A status cannot be detected. For example, although lower frequency of consumption of seafood is associated with a higher risk of low serum retinol concentrations, the seafoods are not quite rich in vitamin $\mathrm{A}$ and thus these correlations are probably due to other food intake factors associated with wealth.

Funding: This study was supported by grants from UNICEF (No. YH101-13).

Ethical approval: This study was approved by the Data Inspectorate of China and by the Regional Committee for Medical Research Ethics.

Competing interest: No benefits in any form have been received or will be received from a commercial party related directly or indirectly to the subject of this article.

Contributors: Jiang JX proposed the study, implemented the field work, analyzed the data and wrote the draft. Lin LM proposed the study. Liang GL analyzed the data. Greiner T revised the draft, gave suggestions on the data analysis and directed the draft writing.

\section{References}

1 Aguayo VM, Baker SK. Vitamin A deficiency and child survival in sub-Saharan Africa: a reappraisal of challenges and opportunities. Food Nutr Bull 2005;26:348-355.

2 Kassaye T, Becklake MR, Receveur O, Hanley JA, Johns T. Association between vitamin A status and lung function level in children aged 6-9 years in Wukro Wereda, Northern Ethiopia. Int J Epidemiol 2001;30:457-464.

3 Fischer Walker CL, Black RE. Micronutrients and diarrheal disease. Clin Infect Dis 2007;45:S73-77.

4 Faber M, Benade AJ. Nutritional status and dietary practices of 4-24-month-old children from a rural South African community. Public Health Nutr 1999;2:179-185.

5 Gittelsohn J, Shankar AV, West KP Jr, Ram R, Dhungel C, Dahal B. Infant feeding practices reflect antecedent risk of xerophthalmia in Nepali children. Eur J Clin Nutr 1997; 51:484-490.

$6 \mathrm{HKI} / \mathrm{IPHN}$. Vitamin A status throughout the lifecycle in rural Bangladesh. National Vitamin A Survey 1997-1998. Dhaka, Helen Keller International and Institute of Public Health 
Nutrition, 1999.

7 Gittelsohn J, Shankar AV, West KP Jr, Faruque F, Gnywali T, Pradhan EK. Child feeding and care behaviors are associated with xerophthalmia in rural Nepalese households. Soc Sci Med 1998;47:477-486.

8 WHO, UNICEF. Global Prevalence of Vitamin A Deficiency. Geneva, World Health Organization Micronutrient deficiency Information System. MDIS Working Paper No. 2. WHO/ NUT/95.3, 1996.

9 Sommer A, Davidson FR. Assessment and control of vitamin A deficiency: the Annecy Accords. J Nutr 2002;132:2845S-2850S.

10 Stephens D, Jackson PL, Gutierrez Y. Subclinical vitamin A deficiency: a potentially unrecognized problem in the United States. Pediatr Nurs 1996;22:377-389.

11 State Statistics Bureau of China. Classification of Chinese Health Status [Chinese]. Beijing: China Statistics Publishing House, 1994.

12 Persson V, Greiner T, Islam S, Gebre-Medhin M. The Helen Keller International Food Frequency Method underestimates vitamin A intake where sustained breastfeeding is common. Food Nutr Bull 1998;19:343-346.

13 Chinese Pediatrics Society. The intervention protocol of subclinical vitamin A deficiency [Chinese]. Zhonghua Er Ke Za Zhi 1995;33:201.

14 Tan ZW, Ma GF, Lin LM, Liu YL, Liu CY, Jiang JX, et al. Prevalence of sub-clinical vitamin A deficiency and its affecting factors in 8669 children of China [Chinese]. Zhonghua Yu Fang Yi Xue Za Zhi 2002;36:161-163.

15 Schemann JF, Banou AA, Guindo A, Joret V, Traore L, Malvy D. Prevalence of undernutrition and vitamin A deficiency in the Dogon Region, Mali. J Am Coll Nutr 2002;21:381-387.

16 West KP Jr. Extent of vitamin A deficiency among preschool children and women of reproductive age. J Nutr 2002;132(9 Suppl):2857S-2866S.

$17 \mathrm{WHO} / \mathrm{UNICEF}$. Indicators for assessing vitamin A deficiency and their application in monitoring and evaluating intervention programs. Micronutrient Series, Geneva, World Health Organization. WHO/NUT, 1996.

18 Ramakrishnan U, Hill ID. Assessment and control of vitamin A disorders. J Nutr 2002;132:2947S-2953S.

19 Nestel P, Melara A, Rosado J, Mora JO. Vitamin A deficiency and anemia among children 12-71 months old in Honduras. Rev Panam Salud Publica 1999;6:34-43.

20 Ballew C, Bowman BA, Sowell AL, Gillespie C. Serum retinol distributions in residents of the United States: third National Health and Nutrition Examination Survey, 1988-1994. Am J Clin Nutr 2001;73:586-593.

21 Kurugol Z, Egemen A, Keskinoglu P, Darcan S, Aksit S. Vitamin A deficiency in healthy children aged 6-59 months in Izmir Province of Turkey. Paediatr Perinat Epidemiol 2000;14:64-69.

22 Maziya-Dixon BB, Akinyele IO, Sanusi RA, Oguntona TE, Nokoe SK, Harris EW. Vitamin A deficiency is prevalent in children less than $5 \mathrm{y}$ of age in Nigeria. J Nutr 2006; 136:2255-2261.

23 van Stuijvenberg ME, Kvalsvig JD, Faber M, Kruger M, Kenoyer DG, Benade AJ. Effect of iron-, iodine-, and betacarotene-fortified biscuits on the micronutrient status of primary school children: a randomized controlled trial. Am J Clin Nutr 1999;69:497-503.

24 Rock CL, Thornquist MD, Kristal AR, Paterson RE, Cooper DA, Neuhouser ML, et al. Demographic, dietary and lifestyle factors differentially explain variability in serum carotenoids and fat-soluble vitamins: baseline results from the sentinel site of the Olestra Post-Marketing Surveillance Study. J Nutr 1999;129:855-864.

25 el-Arab AE, Khalil F, Hussein L. Vitamin A deficiency among preschool children in a rural area of Egypt: the results of dietary assessment and biochemical assay. Int J Food Sci Nutr 2002;53:465-474.

Received June 12, 2007 Accepted after revision December 6, 2007 